\title{
Treatment of Precancerous Lesions of the Cervix of the Uterus by Leep: Experience of the Principal Clinic of the Togolese Association of Family Well-Being Planning Center (ATBEF)
}

\author{
Bingo Kignomon M'bortche1*, Baguilane Douaguibe'2, Kossi Edem Logbo-Akey3, \\ Dédé Regina Ajavon ${ }^{3}$, Tina Ayoko Ketevi Ketevi2 ${ }^{2}$, Solim-Biou Djato4, \\ Francis Baramna-Bagou1, Abdoul-Samadou Aboubakari' ${ }^{3}$, Koffi Akpadza² \\ ${ }^{1}$ Clinique Principale de l'Association Togolaise Pour le Bien-Etre Familial (ATBEF), Lomé, Togo \\ ${ }^{2}$ Service de Gynécologie-Obstétrique du CHU Sylvanus Olympio, Lomé, Togo \\ ${ }^{3}$ Service de Gynécologie-Obstétrique du CHU Kara, Kara, Togo \\ ${ }^{4}$ Service de Gynécologie-Obstétrique du CHR Tsévié, Tsévié, Togo \\ Email: *mbortche@yahoo.fr
}

How to cite this paper: M'bortche, B.K., Douaguibe, B., Logbo-Akey, K.E., Ajavon, D.R., Ketevi, T.A.K., Djato, S.-B., Baramna-Bagou, F., Aboubakari, A.-S. and Akpadza, K. (2021) Treatment of Precancerous Lesions of the Cervix of the Uterus by Leep: Experience of the Principal Clinic of the Togolese Association of Family Well-Being Planning Center (ATBEF). Open Journal of Obstetrics and Gynecology, 11, 1675-1684. https://doi.org/10.4236/ojog.2021.1112157

Received: November 8, 2021

Accepted: December 14, 2021

Published: December 17, 2021

Copyright $\odot 2021$ by author(s) and Scientific Research Publishing Inc. This work is licensed under the Creative Commons Attribution International License (CC BY 4.0).

http://creativecommons.org/licenses/by/4.0/ (c) (i) Open Access

\begin{abstract}
Objectives: Describe the socio-demographic characteristics, describe the main indications for LEEP and present the main complications. Methodology: This was a cross-sectional and descriptive study with consecutive recruitment of the study population through cervical cancer screening campaigns throughout the country during the period July 1, 2017 to April 30, 2019. Included were all patients eligible for LEEP and having benefited from this therapeutic method during our study period. Data were collected from a registry and recorded on a questionnaire developed for this study. These data were analyzed using Epi info 3.5.1 software. The following parameters were studied: patient age, indication for LEEP, intraoperative and postoperative complications, histological examination of the specimens, and postoperative surveillance and screening follow-up one year after LEEP. Results: During the study period, 12,595 women were screened for precancerous cervical lesions. A total of 474 women had precancerous lesions. Of these women, 227 had undergone loop resection, a rate of $47.9 \%$. The main indications for LEEP were extensive lesions (68.7\%), lesions penetrating the internal cervical os (12.8\%). Incidents occurred in $7.5 \%$ of patients during the procedure. Post-operative complications occurred in $14.7 \%$ of cases. Conclusion: LEEP is a better way to treat precancerous lesions but is not well known by medical staff. The equipment of health facilities and the training of medical staff will make it possible to popularize the practice throughout the country. This extension will contribute to the fight against cervical cancer.
\end{abstract}




\section{Keywords}

Precancerous Lesions, Cervical Cancer, LEEP

\section{Introduction}

Cervical cancer is a public health problem, caused in the majority of cases by the human papillomavirus (HPV) contracted most often during sexual practices [1]. Cervical cancer is the fourth most frequently diagnosed type of cancer in women and the fourth leading cause of cancer death in women worldwide [1]. In 2018, there were an estimated 570,000 new cases of cervical cancer and 311,000 deaths from this disease worldwide [1]. In Togo, according to hospital statistics, it is the 2nd most frequently diagnosed cancer in women and the 2nd leading cause of cancer death in women. This mortality rate related to this cancer is due to the delay in diagnosis and the inadequate technical platform in our country. The fight against cervical cancer is based primarily on vaccination and screening for precancerous lesions. Visual inspection of the cervix after application of acetic acid and lugol (VIA/VILI) is a means of screening for precancerous lesions of the cervix in developing countries. Following screening, several techniques are available for the treatment of precancerous cervical lesions. These include cryotherapy, colonization, laser vaporization, and loop electrosurgical excision procedure (LEEP). The latter method has the advantage over the others of providing a specimen for histological study. This removal prevents precancerous lesions from progressing to cervical cancer. Residual or recurrent lesions after LEEP are also rare [2] [3].

Thus, ATBEF, in an effort to reduce the incidence of cervical cancer in Togo, has been offering since June 2017 with the support of the Japanese government through the JTF "Japan Trust Fund" project, screening and management services for precancerous cervical lesions by cryotherapy and diathermy loop resection in the chief towns of the country's six health regions.

This study reviews the management of precancerous lesions by LEEP with the following objectives:

- Describe the socio-demographic characteristics.

- Describe the main indications for LEEP.

- Present the main complications.

\section{Methodology}

This was a cross-sectional and descriptive study with consecutive recruitment of the study population through cervical cancer screening campaigns throughout the country during the period from July 1, 2017 to April 30, 2019, i.e., a duration of 22 months.

The working materials were mainly acetic acid, Lugol's, cryotherapy and diathermy loop resection equipment, cervical biopsy forceps and equipment for 
performing uterine cervical smears. Women aged 20 to 50 years were screened with the VIA/VILI technique. Women who were older than 50 years of age received a cervical smear.

The VIA test was positive when blanching occurred at the squamocolumnar junction (SCJ) or at the transformation zone. The Lugol's test was considered positive if a zone of non-iodine impregnation was noted at the transition zone (iodine-negative zone). Whenever the acetic acid test and/or the iodine test was positive, the patient was managed with cryotherapy or resection if the lesion was large. Resection specimens and uterine cervical smear slides were sent for histopathological examination. Abnormal cervical smears were managed by loop electrosurgical resection. An abnormal cervical smear was considered to be any smear that showed low-grade or high-grade squamous intraepithelial lesions or cervical condylomas.

All patients who were eligible for LEEP and who received this treatment method during our study period were included.

There were three basic steps in LEEP: preLEEP counseling to reassure the patient, collect information about her, provide information about the LEEP procedure, and obtain her informed consent for LEEP; the actual LEEP procedure; and post LEEP counseling to reassure the patient again, discuss normal signs and complications after LEEP, and provide hygienic measures to follow. Once all these steps were completed, the resection specimen was sent with a report card for pathological study. After the procedure, the next appointments were scheduled at 3 months, 6 months and 1 year. However, the patient could return earlier if she showed abnormal signs. Abnormal signs after LEEP included pelvic pain, heavy genital bleeding, persistent genital bleeding, and fetid leukorrhea. The data collection, including the LEEP report, was based on a register developed for this purpose. From this register, the data were collected on a questionnaire developed for this study. These data were analyzed using the Epi info 3.5.1 software. The following parameters were studied: age of the patients, indication for LEEP, intra- and postoperative complications, histological study of the parts, and postoperative surveillance and screening one year later. The cost of VIA/ VILI screening was 2000 CFA francs or US $\$ 4$; the cost of management by loop electrosurgical resection including management of complications was 25,000 CFA francs or US\$50. The ethical consideration taken into account was the respect of anonymity and confidentiality. The consent of the patients was also obtained.

\section{Results}

\subsection{Incidence}

During the study period, 12,595 women were screened for precancerous cervical lesions. Of these, 12,261 women were screened for precancerous lesions by VIA/VILI and 334 women were screened by Pap smear. A total of 474 women had precancerous lesions, representing a frequency of $3.76 \%$. Of these women, 227 had undergone loop electrosurgical resection, representing a rate of $47.9 \%$ of 
lesions (Table 1).

\subsection{General Characteristics}

The table below describes the macroscopic appearance of the cervix before screening, the results of VIA/VILI screening, and the characteristics of precancerous lesions (Table 2).

\subsection{Characteristics of the Cases Managed by Loop Electrosurgical Resection}

The mean age of the patients was 46 years with extremes of 22 and 60 years. The most represented age group was 35 - 40 years in $48 \%$ of cases. The average parity was 3 children with extremes of 0 and 8 children. About $24.7 \%$ of the patients were living with HIV. The average age at first sexual intercourse was 17 years with extremes of 15 years and 27 years (Table 3 ).

\subsection{Treatment of Precancerous Lesions}

\subsubsection{Indications}

Loop electrosurgical resection was performed for various indications (Table 4).

\subsubsection{Incidents during the Procedure}

Nearly $7.5 \%$ of patients had experienced incidents during the procedure. These incidents were: ten cases of pelvic cramps during LEEP, two cases of lipothymia just after the cervical administration of injectable xylocaine, three cases of bleeding due to accidental injury of the vaginal wall, two cases of difficulty in haemostasis due to failure of the electric generator.

\subsubsection{Operative Follow-Up after LEEP}

We recorded $14.7 \%$ of complications after LEEP, such as pelvic pain $(39.4 \%)$,

Table 1. Frequency of precancerous lesions of the cervix.

\begin{tabular}{lcc}
\hline & Number & Percent \\
\hline Screening Technique $(\mathrm{n}=12,595)$ & & \\
VIA/VILI ${ }^{*}$ screening & 12,261 & 97.3 \\
Screening by Pap smear & 334 & 2.7 \\
Cases requiring management $(\mathrm{n}=474)$ & & \\
Positive VIA/VILI test & 451 & 45.1 \\
Abnormality on Pap smear & 23 & \\
Managed (n = 474) & & 52.1 \\
Managed with cryotherapy & 247 & 47.9 \\
Managed with LEEP & 227 & \\
\hline
\end{tabular}

${ }^{\star}$ VIA: visual inspection with acetic acid; ${ }^{\star}$ VILI: visual inspection with lugol; Cervicouterine smear. 
Table 2. General characteristics of screening results.

\begin{tabular}{lcc}
\hline & Number & Percent \\
\hline Macroscopic aspect of the cervix $(\mathbf{n}=\mathbf{1 2 , 5 9 5})$ & 12012 & 95.4 \\
Pink cervix & 72 & 0.6 \\
Red cervix & 56 & 0.4 \\
Cervix bleeding on contact & 455 & 3.6 \\
Cervicitis & & \\
VIA and VILI test ( $\mathbf{n}=\mathbf{1 2 , 2 6 1 )}$ & 451 & 3.7 \\
Positive test & 11810 & 96.3 \\
Negative test & 247 & 54.8 \\
Characteristics of white acetowhite or iodine-negative lesions on test (n & $=451)$ \\
Small lesion eligible for cryotherapy & 29 & 6.4 \\
Lesions penetrating the internal os & 16 & 3.5 \\
Extensive lesion beyond 75\% of the involved cervix & 156 & 34.6 \\
Extent of lesion exceeds cryoprobe surface. & 3 & 0.7 \\
Lesions persisting one year after cryotherapy & & \\
Histology of the Pap smear (334) & 223 & 66.8 \\
Normal examination & 88 & 26.3 \\
Cervicitis & 11 & 3.3 \\
low grade squamous intraepithelial lesions & 9 & 2.7 \\
high-grade squamous intraepithelial lesions & 3 & 0.9 \\
Condylomatous lesion of the cervix & & \\
\hline
\end{tabular}

Table 3. Characteristics of the cases managed by loop electrosurgical resection.

\begin{tabular}{|c|c|c|}
\hline & Number & Percent \\
\hline \multicolumn{3}{|c|}{ Age in years $(n=227)$} \\
\hline$[20-25[$ & 3 & 1 \\
\hline$[25-30[$ & 5 & 2 \\
\hline$[30-35[$ & 15 & 7 \\
\hline$[35-40[$ & 109 & 48 \\
\hline$[40-45[$ & 23 & 10 \\
\hline$[45-50[$ & 67 & 30 \\
\hline$\geq 50$ & 5 & 2 \\
\hline \multicolumn{3}{|l|}{ Parity $(n=227)$} \\
\hline Nullipare & 12 & 5 \\
\hline $1-4$ & 164 & 78 \\
\hline $4-8$ & 51 & 22 \\
\hline \multicolumn{3}{|c|}{ HIV status $(n=227)$} \\
\hline HIV positive & 56 & 24.7 \\
\hline HIV negative & 171 & 75.3 \\
\hline \multicolumn{3}{|c|}{ Age in years at first intercourse $(n=227)$} \\
\hline$[15-20[$ & 123 & 54 \\
\hline$[20-25[$ & 85 & 37 \\
\hline$[25-30[$ & 19 & 8 \\
\hline
\end{tabular}


significant genital bleeding (24.2\%), infection of the resection site (15.2\%) and persistent minimal genital bleeding (21.2\%) (Table 5).

\subsubsection{Monitoring Results}

Patients were followed up at three months, six months and twelve months after treatment.

The monitoring results were as follows: 3 months, 6 months and one year

- At 3 months: all cervixes examined were well healed.

- At 6 months: no particularities found on examination.

- At 1 year: we had lost sight of 17 patients. All the women examined had a healthy cervix. Two women had an acetowhite lesion at the follow-up screening. They had undergone a total hysterectomy.

\subsection{Histology}

Histological findings according to the Bethesda classification showed that low grade intraepithelial lesion represented $45.4 \%$ of cases (Table 6).

Table 4. Indications for LEEP.

\begin{tabular}{lcc}
\hline & Number & Percent \\
\hline Lesions penetrating the internal os & 29 & 12.8 \\
Lesion extended beyond 75\% of the involved cervix & 16 & 7.1 \\
Extensive lesion exceeds cryoprobe surface. & 156 & 68.7 \\
Low grade squamous intraepithelial lesions & 11 & 4.8 \\
High grade squamous intraepithelial lesions & 9 & 4 \\
Condylomatous lesion & 3 & 1.3 \\
Lesions persisting one year after cryotherapy & 3 & 1.3 \\
Total & 227 & 100 \\
\hline
\end{tabular}

Table 5. Complications following surgery and their management.

\begin{tabular}{lcc}
\hline & Number & Percent \\
\hline Postoperative complications $(\mathrm{n}=33)$ & 13 & 39.4 \\
Pelvic pain & 8 & 24.2 \\
Significant bleeding & 5 & 15.2 \\
Fetid discharge & 7 & 21.2 \\
Persistent minimal bleeding & & \\
Management (n = 34) & 15 & 44.1 \\
Re-establishment of cervical hemostasis & 5 & 14.7 \\
Antibiotic treatment & 1 & 2.9 \\
Blood transfusion & 13 & 38.3 \\
Analgesic treatment & & \\
\hline
\end{tabular}


Table 6. Histological findings according to the Bethesda classification.

\begin{tabular}{lcc}
\hline & Number & Percent \\
\hline Low-grade intraepithelial lesions & 103 & 45.4 \\
High-grade intraepithelial lesions & 61 & 26.9 \\
Carcinoma in situ & 2 & 0.9 \\
Flat condyloma & 32 & 14.1 \\
Normal examination & 29 & 12.7 \\
Total & 227 & 100 \\
\hline
\end{tabular}

\section{Discussion}

\subsection{Frequency}

The frequency of ARD during our study period was $47.9 \%$. This rate is higher than those of Millogo et al. [4] in Burkina Faso who reported a frequency of 4.2\% and lower than Millogo et al. in 2017, who reported 54.9\% [5]. This high rate found in our study could be explained by the characteristics of the lesions since in $34.6 \%$ of cases, the extent of the lesion exceeded the surface of the cryoprobe.

\subsection{Indications}

In our series, the indications for LEEP were lesions penetrating the internal os of the cervix, extensive lesions beyond $75 \%$ of the involved cervix, lesion extent exceeds the cryoprobe surface, lesions persisting one year after cryotherapy.

According to Millogo et al. in 2017 [5], the main indications were large acetowhite lesions, abnormalities on biopsy, bleeding cervix on contact, large ectropion (6.2\%), cervical polyp, questionable VIA/VILI results (4.6\%), abnormalities on FCV (3.8\%), persistent lesions after other treatments (1.5\%). For Monsonego [6], the indications for excisional treatments such as LEEP are: CIN 2 or 3, in situ appearance, CIN 1 with a non-visible junction, inadequate colposcopies with a high-grade smear, suspected or even confirmed micro-invasive cancer on biopsy, as this is the only way to accurately measure the depth of invasion below the basal area, on which depends the course of action.

Some authors [7] believe that it is not always necessary to treat a precancerous lesion. Depending on its severity, two options are possible: monitor the lesion until it heals spontaneously or remove it. On the other hand, other authors, such as Lewis et al. [8], believe that screening is of no use if it is not followed by the implementation of an effective treatment in those who have the disease. We agree with these authors that screening will not be useful if it is not followed by effective treatment. In our context, surveillance is often problematic because of the approach to screening for precancerous lesions, since screening is done by a team during campaigns. The date of the next campaigns in the same settings is not known in advance. As the screening service is not available in the health facilities, it would be difficult to institute this periodic monitoring of positive cases 
in order to make a judgment on the evolution or regression of the lesions. This situation allowed us to systematically propose treatment when the diagnosis of a precancerous lesion was made. The fight against cervical cancer will be carried out through awareness-raising activities, the diversified availability of means of treating precancerous lesions in the country's various health facilities and the active involvement of the various stakeholders. Cryotherapy and thermal ablation are other types of treatment when the precancerous lesion occupies less than $75 \%$ of the surface of the cervix and does not penetrate the cervical canal.

\subsection{Incidents}

During the resection three types of incidents occurred: ten cases of pelvic cramps during LEEP, two cases of lipothymia just after the cervical administration of injectable xylocaine; three cases of bleeding by accidental injury of the vaginal wall, two cases of difficulty in haemostasis due to the failure of the electrical generator. It should be noted that cases of lipothymia are normal events that last only one to two minutes and may vary from person to person [9]. This underscores the importance of counseling prior to LEEP. This situation allowed us to use $1 \%$ lidocaine instead of the $2 \%$ previously used. The accidental vaginal lesions could be explained by the lack of mastery of the procedure at the start of this management. These incidents could be avoided by caution during resection and the effect of continuous learning. The failure of the generator was found to be an incident before hemostasis was performed. This situation makes it possible to always have a backup generator during the campaigns and to perform preventive maintenance on these generators.

Millogo et al. in 2017 [5] found pelvic pain in the form of lower abdominal cramps, followed by bleeding and secondary manifestations of lidocaine as incidents in his series.

Some authors [9] recommend that patients take two tablets of a non-aspirin analgesic such as ibuprofen or acetaminophen to prevent abdominal cramps.

\subsection{Complications and Their Management}

We recorded $14.7 \%$ of complications after LEEP, such as pelvic pain (39.4\%), significant genital bleeding (24.2\%), infection of the resection site (15.2\%), and minimal persistent genital bleeding (21.2\%).

Our rates are largely lower than those of Millogo et al. in 2017 [5] who had recorded in their series, $63.8 \%$ cases of complications after LEEP. Cases of minimal bleeding constituted $47 \%$, profuse bleeding $26.5 \%$, infections of the resection area $26.5 \%$.

Schiavone [10] and Alexandre [11] note respectively $3 \%$ of cases of cervical stenosis, which we did not record in our series, and obstetrical complications, which were not the subject of our study. According to the WHO [12], hemorrhage can be diffuse or of arterial origin. Infections in the resection area have several causes: resumption of sexual intercourse too early, poor intimate hy- 
giene, vaginal cleansing with products that are aggressive for the vaginal flora. The management of these complications is necessary, as their presence could constitute an obstacle to the proper healing of the surgical wound.

In our series, all the patients who presented a complication had received treatment. Haemostasis had been resumed by coagulation for the patients who presented minimal or abundant genital bleeding. Medical treatments based on antibiotics and antiseptic ova were administered in patients who had a resection site infection. A transfusion of two bags of packed red blood cells was administered to a patient who was hemodynamically unstable after significant genital bleeding.

\subsection{Monitoring}

In our series, patients were followed up at 3 months, 6 months and 1 year. The WHO [12] recommends the first follow-up visit 2 to 6 weeks after LEEP, followed by further visits at 6 and 12 months. Other authors [9] recommend a follow-up examination every 6 to 9 months after treatment. Baldauf et al. [13] recommend the first visit between 3 and 6 months after lesion removal, a second between 9 and 15 months and a third between 24 and 36 months. The screening at one year had found the precancerous lesion. This situation allowed us to perform a total hysterectomy in these two women. It is important to note the interest of this systematic control screening one year after the initial treatment.

\section{Conclusion}

The management of precancerous lesions of the cervix by LEEP is effective in Togo. It allows to obtain a complete cure and to appreciate the quality of the screening by the histological confirmation of the resection parts. Thus, LEEP is a better way to treat precancerous lesions. ARD is not well known among medical personnel. Equipping health facilities and training medical staff will allow the practice to be popularized throughout the country. This popularization of the practice will contribute to the fight against cervical cancer.

\section{Conflicts of Interest}

The authors declare no conflicts of interest regarding the publication of this paper.

\section{References}

[1] Bray, F., Ferlay, J., Soerjomataram, I., Siegel, R.L., Torre, L.A. and Jemal, A. (2018) Global Cancer Statistics 2018: GLOBOCAN Estimates of Incidence and Mortality Worldwide for 36 Cancers in 185 Countries. CA: A Cancer Journal for Clinicians, 68, 394-424. https://doi.org/10.3322/caac.21492

[2] Alliance for Cervical Cancer Prévention (ACCP) (2003) Méthodes de dépistage visuelles des alternatives prometteuses. Prévention du cancer cervical: Aide mémoire. https://www.path.org/publications/files/RH_visual_screening_fs_fr.pdf

[3] D’halluin, G. (2018) Laser du col de l'utérus. La chirurgie gynécologique et mam- 
maire de charente.

http://www.chirurgie-gynecologie.fr/page-chirurgie-gyn/laser-col.html

[4] Millogo, F.T., Akotionga, M. and Lankoandé, J. (2004) Dépistage du cancer du col utérin dans un district sanitaire (Burkina Faso) par biopsie de volontaires après application d'acide acétique et du lugol. Bulletin de la société de pathologie exotique, 97, 135-138.

[5] Millogo Traoré, T.F.D., Ouattara, A., Hounsounou, I.V.S. and Nebie, P.S. (2017) Prise en charge des lésions précancéreuses du col utérin par la résection à l'anse diathermique au Centre Hospitalier Universitaire Yalgado Ouédraogo: bilan de huit mois d'activité. Journal de la $S A G O, 18,27-33$.

[6] Monsonégo, J. (2006) Infections à papillomavirus: État des connaissances, pratiques et préventions vaccinales. Springer, Paris. http://www.myilibrary.com

[7] Ndoua, C.C., Tebeu, P.M., Kemfang, J.D. and Kasia, J.M. (2015) Evaluation des résultats après traitement des lésions intra épithéliales du col utérin par la cryothérapie: étude préliminaire au Centre Hospitalier Universitaire de Yaoundé: A propos de 21 cas. Pan African Medical Journal, 20, Article No. 225. https://doi.org/10.11604/pamj.2015.20.225.4618

[8] Lewis, K.D.C., Sellors, J.W. and Dawa, A. (2011) Report on a Cryotherapy Service for Women with Cervical Intraepithelial Neoplasia in a District Hospital in Western Kenya. African Health Sciences, 11, 370-376.

[9] Greenberg, S.E. (2005) Traitement "LEEP” (excision électro-chirurgicale avec anse diathermique). Campus Riverside, Clinique externe de gynécologie/oncologique de L'Hôpital d'Ottawa, 9 p.

[10] Schiavone, S. (2011) Grossesse après conisation: étude transversale des conséquences obstétricales de la conisation sur la grossesse réalisée à la Maternité Régionale Universitaire de Nancy entre 2002 et 2010. Sciences du Vivant. https://hal.univ-lorraine.fr/

[11] Alexandre, L. (2014) Le suivi des CIN après une conisation. Université de Rennes 1, France, $18 \mathrm{p}$.

[12] Organisation Mondiale Santé (2007) La lutte contre le cancer du col de l'utérus. Guide des pratiques essentielles. Organisation Mondiale Santé, Genève, 287 p.

[13] Baldauf, J.J., Ritter, J., Cuenin, C., Dreyfus, M., Elmokaddam, Y. and Walter, P. (1999) The Therapeutic Results of Conization with Diathermic. Contraception Fertilité Sexualité, 27, 140-146. 